\title{
Richtige Cebensfïhrung
}

Uolkstümliche Huf[ätse zur Erziehung des Menjaben nach) den Grundlätsen der Individualplychologie Gerausgegeben von sofie Lazarsfeld

\section{dielesbe?teflumaen}

"11 iere" satöruแne॥

Uon

Dr. Flfred Hdler

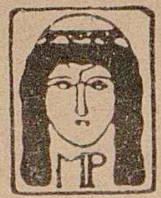

3 wien, und Leipzig a g von moritz perles 40569 



\section{Richtige Cebensiuibrumg}

Volkstümliche Huflätze zur Erziebung des Men[(b)en nac) den Grund[äżen der Individualplychologie 5erausgegeben von Sofie cazarsield

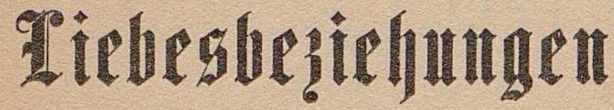

แ11i Deren Gitörutugen Jon

Dr. Hifred मdier

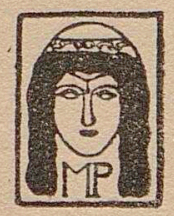

1920

wien und Leipzig

Jerlag von moritz perles Wien, I., Seilergalle 4. 

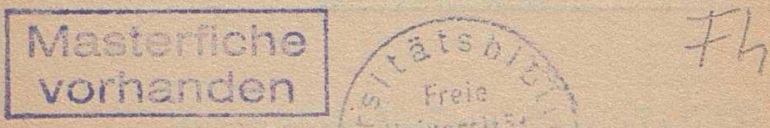

Hile Rechte, ein(chlieblich) des Rechtes det ẗberletzung in fremde Sprachen, vorbebalten. 
Um einen menichen ganz kennen zu lernen, ilt es not= wendig, ibn auch in [einen Ciebesbeziehungen zu veritehen ... velir millien von ibm auslagen können, ob er (ich) in $\mathrm{Hn}=$ gelegenbeiten der liebe richtig oder unrichtig verbält, wir müllen feltitellen können, wa rum er in einem fall geeignet, im anderen Falle ungeeignet ift oder lein wïrde. Es gelellt (id) uns dann ganz von lelbit die weitere Hufgabe: wie wir es denn an[tellen könnten, in Ciebesbeziebungen irgendweldbe Feblichläge zu verbindern. (Uenn man bedenkt, dab ja von der lölung des Ciebes = und Eheproblems vielleicht der größzte Ceil des men[chlichen Glückes abbängig i[t, wird uns lofort klar, da $\mathfrak{B}$ wir eine Summe der alleribweritwiegenden Fragen vor uns baben.

Eine Schwierigkeit taucht bei Erörterung dieler Fragen gleich) anfangs auf - die meiften [prechen lie lofort aus. Die menlden leien nicht alle gleich und vielleicht hätten zwei Menichen unter anderen Umitänden glüdklicher lein können, wenn beilpielsweile jeder einen anderen Partner ge= funden hätte. Diele Möglichkeit loll gerne zugegeben werden, lie lagt jedoch nichts anderes aus, als daj die Betroffenen eine [chlechte $(U / a b)$ getroffen haben. $0 b$ das Scheitern am Liebesproblem in der [chlechten, irrtümlichen (Uab] zu [uchen ilt, oder ob wir Fälle betrad)ten, in denen einer unter allen ulm[tänden am Ciebesproblem ge[cheitert wäre, weil er aus tieferen Gründen [cheitern $m u \not B t e$, in vielen Fällen wird uns die Renntnis der men[dhlichen Seele und ibrer bewegenden Kräfte vor Febli(hlägen bewabren können. 
Die Frage der Liebesbeziebungen ift eine Ceilfrage des men[d)lid)en Cebens. Ibr Uer[tändnis ift nur möglid, wenn wir den Zulammenbang mit allen übrigen Lebensfragen be= achten. Das Ceben [tellt uns drei grobe Hufgabenkomplexe, von deren cölung unlere Zukunft, unler Cebensglïck ab= bängig ilt.

Die erite Cebensaufgabe ift die ge[ell] chaftliche Huf= gabe im weiteiten sinne. Das Ceben verlangt von jedem ein beltimmtes Uerbalten und eine weite[tgehende Kontaktfäbigkeit unieren nebenmen[chen gegenüber, ein beftimmtes Uerbalten inmerhalb der Familie und eine formulierung leiner [ozialen Einjtellung. Es i[t für das Schick[al eines Men[chen nicht gleichgültig, nach welcher Ridbtung er (id) zum Beilpiel eine loziale Ordnung zum richtunggebenden Ziel lettt, wieweit er bei [einen நandlungen an das eigene $(20)\}$ und wieweit er an das $\left.\left(U_{0}\right)\right]$ der anderen denkt. Seine innere $(Z J a b]$ i[t bierbei in leinen äu[seren Ent[chlül[en oft [d)wer zu finden, oft karn er (ich) in Fragen einer [ozialen Stellungnabme ïber= baupt nid)t ent[deiden, und oft ift lein Standpunkt in anderem Sinne zu ver[tehen, als es äujerlich den Hn[chein hat. Huch von der politi[chen Stellungnabme gilt äbnliches. Selten trifft man Men[ahen, die mit ibrer Partei zufrieden lind, febr oft aber (olche, die man eigentlich einer anderen Partei zurechnen möchte. Immer [pielt ihr Uerbalten zur men[(h)lichen Gemein= [chaft, ibr Uerbalten zum nebenmen[chen im weite[ten Sinne die gröbte Rolle, nicht, was er oder andere davon denken. Gine weitere Cebensaufgabe, die unlerer Lölung harrt, ift die Berufsfrage, das ift die Art und tueile, wie einer leine Kräfte der Hllgemeinheit dien[tbar machen will. Die cölung diefer Frage beleuchtet auberordentlich (charf das tulejen eines Menjhen. tUenn wir zum Beifpiel von einem jungen Manne bören, dab ibn jeder Beruf anwidert, to werden wir ibn vorer[t nicht für einen geeigneten Mitmen[chen balten, entweder weil er für die Gelellichaft noch nicht reif i[t oder weil er obne Belehrung von (elbit auch gar nicht reif werden wird. Zur Ergreifung eines Berufes fübren unbewu[3te Zu= \{ammenhänge, die bei der weit überwiegenden Mebrzab] der 
menichen eingreifen. Unbewußst find die[e Zulammenhänge, weil niemand, wenn er einen Beruf ergreift, daran denkt, daß 3 er einen Schritt zum nutsen der Allgemeinheit unter= nommen, dak er (id) einen Plats in der allgemeinen Hrbeits= teilung gefucht bat. Freilich kommt es weiters auch darauf an, wie er (ich) in leinem Beruf betätigt. Es gibt ja men[chen, die wohl bis zu einer Berufswabl gelangen, innerbalb ibres Berufes aber ver[agen oder nach einiger Zeit erkennen, [ie bätten eigentlich etwas anderes werden follen. (Ulir werden aus einem bäufigen (J)echlel der Berufe [thlieben, dak wir Menj(ben vor uns baben, die eigentlich gar keinen Beruf baben möchten, (ich vielleicht fuir jeden Beruf als zu gut, allo zu [chlecht dünken und nur fo tun, als ob lie mitgingen. Die dritte Cebensfrage, die jeder men/ch lölen muj, ift nun die Ciebes= und Ehefrage, die wir bier belonders betrachten wollen. In die(e Frage wäch) lt das kind nach) und nach hinein. Seine ganze Ulmgebung ift erfüllt von Liebes: und Ehebeziebungen. Es ift nicht zu verkennen, dajs das Rind [bon in den allerer[ten Cebensjabren zu dieler Frage Stellung zu nebmen und (ich) eine Richtung zu geben ver= [ucht. ZUas wir von dieler Catlache in tJorten bören, ift nicht enticheidend, denn lobald es auf Liebesfragen zu [prechen kommt, bemäd)tigt (iic) leiner oft eine ungebeure Scheu. Es gibt Kinder, die es ganz dezidiert ausiprechen, da仍 lie über dieles Chema "nicht reden können. Es gibt Kinder, die ibren Eltern lebr zugetan (ind, es aber nicht zultande bringen, mit ibnen zärtlich zu lein. Ein vierjähriger knabe bat Külle, die man ibm geben wollte, mit Schlägen ins Gelicht beant= wortet, weil ibm das Gefühl einer zärtlichen Regung un= beimlich war, ihm beängltigend, geradezu demütigend fchien. Huch) bei einem Rückblick in unler eigenes [eben wird es uns nicht entgehen können, daj jede Zärtlid)keitsregung von einer Hrt Schamgefüb] begleitet wird und von dem Eindrucke, als würde man dadurch (ch)wächer werden oder im tuerte finken. Dies ift lebr merkwürdig und bedarf einer Erklärung. (Uir wach)en in der Stimmungslage auf, als ob eine zärtliche Regung eine Schande wäre. Diele Stimmungslage ftimmt 
überein mit der einheitlichen Richtung unjerer Kultur nach) einem männlichen Ideal. Dementiprechend werden unjere Kinder in Schule, Citeratur und jeder Umgebung fortwäbrend in der Richtung erzogen, in der Ciebe eine Hrt (lnmännlicbkeit zu erblicken, und fie drücken das manchmal auch deutlic) aus. Manche gehen darin 10 weit, daf man von ibnen als von gefübls [ he uen Men[chen iprechen kann.

Die er[ten Zärtlicb)eitsregungen des kindes treten [chon lehr frübzeitig in Er[cheinung. (Jir können aus der Entwick= lung der[elben [elor leicht fe[titellen, dak fie alle Regungen

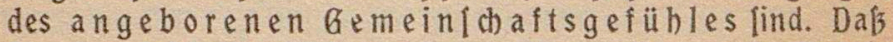
das Gemein[(h)aftsgefübl angeboren ift, erbellt aus der Regel= mäßjigkeit [eines jedesmaligen Fuftretens. Der Grad [einer Ent= faltung gibt uns die Möglichkeit, die Stellung zum Leben zu überblicken. Im Begriff "Men[(c)" liegt bereits un[er ganzes Uer[tändnis für das Gemein[(h)aftsgefübl, wir könnten uns einen menichen, der es verloren bätte und dennoch als Menl(h) be= zeichnet werden [ollte, nicht voritellen. Huch in der Ge[chichte finden wir i[oliert lebende Men[aben nicht. (UJo immer Men[d)en angetroffen wurden, fand man lie in Gruppen vor, wenn die einzelnen Men[(h)en nicht etwa kün[tlich oder durch tUabn[inn voneinander getrennt waren. Darwin weilt im Cierreiche nad), dak jene rebewelen in Gruppen leben, die der Natur gegenüber eine ungün|tigere Pofition haben. Die Uitalität, die Cebenskraft diejer ungün|tiger geftellten Ciere wirkt (ich) dahin aus, daj dieje Ciere (ich zu Gruppen zu[ammen[d)lieken, un= bewulst einem Prinzip der Selb[terbaltung folgend. Zdir können ferner veriteben, daj alle jene vereinzelt lebenden Ciere, denen in ibrer [tiefmütterlichen Husbildung das Gemein[d)aftsgefüb] gemangelt hat, zugrunde gehen mußsten. Sie fielen einer natür= lichen Huslele zum Opfer. Das Prinzip der natürlichen Huslefe ift auch dem Menichen gegenüber gefäbrlich), da er der natur gegenüber körperlich am (tiefmuitterlich[ten ausge[tattet i[t.

Die Situation der Minderwertigkeit und Unzulänglichkeit des men[कhenge[chlechtes entwickelt im ganzen und im einzelnen einen fortwäbrenden Hntrieb und Zwang, der uns to lange forttreibt, bis ein ungefäbrer Rubezu[tand erreicht ift und ein Beftand 
gelichert ericheint. Huf diejem tuege befinden wir uns auch jetst noch, und es i[t heutzutage vielleicht der [chön[te Croit des men[chen, wenn er (ich) bewukt ift, daß unlere beutige Situation nichts anderes ift als ein Durdh gangspunkt, eine augenblickliche Pbaje men[chlid)er Entwicklung. Ihn wird in allen Fragen des Cebens natürlich der am beften durch)(c)reiten, der mit den tat[äcblichen Uerbältnil[en in Einklang [teht, der der Cogik der Catfachen gerecht wird, während naturgemäß ein unerbittliches bej(bick diejenigen trifft, die (ich) diejer Logik wider[etsen. Im tiefften Sinne aber i[t das Gefübl für die [ogik des men[(h)lichen Zulammenlebens $G$ e me in (c) a fts $g$ ef übl.

Die ganze Entwicklung des kindes verlangt dellen Ein= bettung in eine Situation, in der Gemein|(h)aftsgefühl vorban= den ift. Sein Leben und leine Ge[undheit find nur dann ge= wäbrleiltet, wenn Menld)en vorbanden (ind, die (ich) für das kind einletsen. Ein neugeborenes Kalb kann zum Beilpiel (d)on in kurzer Zeit Giftpflanzen von anderen unter[cheiden. Der neugeborene Men(c) jedoch ift infolge der Minderwertigkeit [eines Organismus auf das Gemein[(h)aftsgefüb] der Erwach) [enen angewielen, man muj das kind lange betreuen, belebren und erziehen, bis es [elb[t die Fäbigkeiten erwirbt, (iid) zu erbalten.

Huch) wenn wir die Fäbigkeiten betrachten, die unleren Stolz ausmachen und uns den Uorrang vor anderen Lebewelen [ichern, wie Uermunft, Logik, die Sprache, un[er Uer[tändnis und unjere Uorliebe für alles Schöne und Gute, fo können wir in ihnen auch nur Uorzüge erblicken, die der einzelne menf(h) nie bätte bervorbringen können, weil [ie gewiflermaken erit aus einer Mallenleele geboren werden konnten. Ulir be= friedigen damit Bedürfnille, die den einzeinen nie bedrüdkt hätten, die erit in einer men[chlichen Gemein[chaft lebendig wurden. Für einen e in z e I ne $n$ Men[chen, der keinen Zu[amment= bang mit einer Gemein[(b)aft hätte, wäre die bewukte, bewukbt überwachte [ogik gänzlich gleichgültig, er müiste nicht (prechen, es wäre einerlei, ob er gut oder böle i[t, ja dieje Begriffe verlören mangels ibrer Beziebung zu einer men[chlichen $\mathrm{Be}=$ meinf(b)aft, zu einem nebenmen[chen, wie bei einzelnen Cieren, jeden Sinn. Hlle Qualitäten des men(d)lichen Seelenlebens, alle 
Ceiftungen des menlablichen Geiltes lind nur in einem Zul= lammenbang der Menichen untereinander denkbar.

Und für dielen Zulammenthang lorgen nicht nur die not, nicht nur die drängenden Bedürfnifle des Cages, londern auch uniere lexuelle Organilation. Die Ceilung der Men[chbeit in zwei $(e[(d)]$ echter bildet durchaus keine Separation, fondern fie bedeutet einen ewigen Zwang zueinander. Sie gebiert das Gefuibl des gegen[eitigen Uerwandt|eins, weil in den Hdern eines jeden gemeinlames Blut fließst, weil jeder Flei[ch i[t vom Fleild) des anderen. Die Ehegeletse der Uölker find nur unter dem Gelichtspunkt zu ver|tehen, da\} lie die liebe als ein gemeinfames Band der Gruppe betrachteten. Sie verboten daber Ehe[chliebungen und den Sexualverkebr unter Angebörigen einer Familie, weil dies zu einer IJolierung der Familien gefübrt hätte. Dichter, Religionen, die heiligen Gebote wenden (ich) gegen die Inzudt)t und ver[uchen (ie auszutilgen. Die gelebr= teiten Men(d)en baben (ich den Kopf zerbrochen, worin eigent= lich die natürliche Hbwendung der Familienmitglieder gegen= einander begründet [ei. Ulir veritehen [ie ganz leicht auf der Balis des (ich) in jedem Kinde entwickelnden Gemeinl(c)afts= gefühles, das alle möglichkeiten aus(c)altet, die zu einer Ijolirung der Men[chen fübren könnten.

nun find wir (c)on zu jenem Punkte gelangt, von welchem aus wir bereits ver[tehen können, dab das, was wir im eigent= lichen Sinne Lieb e nennen, die Beziehung der (Ge[chlechter, immer mit dem Gemein(chaftsgefüh] zu[ammenbängt und von ibm nicht zu trennen i[t. Die Ciebe als eine Beziebung zu zweit bat als ein Ceil des Gemein[c)aftsgefühls ibre eigenen Geletse und ift als notwendiger Be[tandteil der Erbaltung der men[(b)lichen Gelell[(h)aft aus der Gemeini(d)aft nicht wegzu= denken. (Jer die Gemeinichaft bejaht, bejaht notwendigerweile auch die Liebe. UUer Gemein[chaftsgefühl belitst, muj für die Ehe oder eine ibr gleich = oder böherwertige Form der Liebe eintreten. Derjenige dagegen, de[len Gemein[(haftsgefüb] ge= drollelt ilt, der zu keiner freien Entfaltung leines welens innerhalb der Menichbeit gekommen i[t, wird aud) eine $a b=$ londerliche form leiner Liebesbeziebungen aufweilen. 
Wir können nun zurückblickend eine Fnzab] von Schlül[en zieben, die uns die Überlicht über das grope Gebiet der Ciebesbeziebungen erleid)tern und uns das Dunkel plötlich ein wenig erbellen. Wuir können feflftellen, daj ein Men[ch, dellen loziale Entwicklung Schaden gelitten bat, der keine Freunde bat, kein richtiger mitmenich geworden ift, der eine Uleltan[(h)autung [ein eigen nennt, wie fie dem Gemein[chafts= gefiibl wider\{pricht, der audh vielleidht leine Berufsfrage nicht gut lölen konnte, allo wieder der, der für die Gemein|chaft ganz oder nabezu ganz verloren ilt, in leinen Ciebesbeziehungen Schwierigkeiten baben muḱ, ja kaum im[tande lein wird, die erotilche Frage zu lölen. Die 10 gearteten Men[then werden ablonderliche 2 lege einichlagen, Schwierigkeiten [chaffen und, wo lie (ich ibnen darbieten, wie nach einer (ichernden Hus= rede nach ibnen greifen. Diele Schwierigkeiten wollen wir nun etwas genauer betrachten, wobei wir in das ganze Problem eine tiefere Ein[id)t gewinnen werden. tJir werden fe[titellen können: audh) in den Ciebesbeziebungen eines Men[ (h)en (c) wingt [eine ganze Per[önlichkeit mit. Es i[t uns [owob] möglich, aus [einen Liebesbeziehungen [eine Perlönlicbkeit mitzuveritehen, als auch) aus dem Uer[tändnis [einer Ge[amt= per[önlichkeit beraus die dazu pallende Eigenart leiner ero= tilchen Aniprüche zu erraten.

Überaus bäufig finden wir innerhalb der eroti[chen $B e=$ ziebungen (ehr verbreitete, wenn aud) irrtümliche Uoraus= letzungen, dak Liebe den anderen Ceil verpflichte.

tuenn wir ein wenig ins Leben bineinborchen und uns bierbei ein wenig lelbit beobadten, lo können wir uns davon überzeugen, dak wir lebr oft den Irrtum begehen, zu glauben, die geliebte Perion (ei durch die Cat[ache, geliebt zu werden, bereits verpflichtet. Die[er Irrtum [cheint irgendwie in unlerer ganzen Hnj (b)aungsform enthalten zu lein. Er ftammt aus der Kindheit und aus den Beziebungen innerbalb der Familie, in welcher in der $\tau$ at die liebe des einen nabezu die Uer= pflichtung des anderen ilt. Wir tragen nur einen Reft diejer kindlichen Hnichauung in uns, wenn wir diele Uerbältnille ins Ceben übertragen wollen. Die hieraus entitehenden Hus= 
artungen gruppieren (ich) etwa um den Gedankengang: "(U) il id) dich liebe, mukt du das und das tun." bierdurch kommt auch) oft in Beziebungen von Men[(hen, die (ich) wirklid) zu= getan [ind, ein viel bärterer Ein[chlag binein, und das Machtbedürfnis des einzelnen, der, geftütst auf die Betonung leiner eigenen Liebe, den anderen in lein Schema, in leine Schablone bineinziehen will, fordert, dafs Schritte, Mienen, Gebärden, Lei[tungen etc. lo erfolgen, wie er will, nur aus dem Grunde, "weil er diele Perlon liebt". Dies kann leicht zur Cyrannei ausarten. Eine Spur biervon finden wir vielleidht in jeder Liebesbeziebung.

tuir [ehen lo jenen Faktor das Ciebesleben der Men[chen durch)zieben, der auch (on[t immer zu Störungen des Mit= men[chentums fübrt: das Streben nach Macht und per[önlicher Überlegenheit. In einer men[chlichen Gemein|chaft muks man die Freiheit der per[önlichen Individualität to weit achten, dałs man ibr das freie Ermel[en anbeim[tellt. (U)er nach per[ön= licher Überlegenbeit [trebt, verhindert [einen Hn[chlußs an eine Allgemeinheit. Er will nicht leine Einfügung in das Ganze, londern die Unterordnung der anderen. Damit [tört er natür lich die Garmonie im Leben, in der Gelelifchaft, unter leinen Mitmenichen. Da kein Men[ch lo geartet i[t, dafs er im[tande wäre, (ich dauernd ein Joch auferlegen zu laflen, müllen lolche, die auch in ibren Liebesbeziehungen Macht über den anderen Ceil er[treben, übergrobe schwierigkeiten antreffen. Wollen lie ibre neigung zu überbebung und Überlegenbeit ins eroti[che Problem bineintragen, 10 müllen lie (ich) entweder einen Partner [uchen, der (ich) ibnen [cheinbar unterwirft, oder aber, lie nebmen den Kampf mit einem Partner auf, der ebenfalls in der Erotik überlegenheit oder Sieg lucht oder dazu verleitet wird. Im eriten fall erleben wir die Umwand= lung der Ciebe in Sklaverei, im zweiten Fall [eben wir einen dauernden, gegenleitig aufreibenden kampf um die Macht voraus, der nie irgendwelche Garmonie veripricht.

Die Zlege, die hierbei belchritten werden, lind auber= ordentlic) mannigfach. Es gibt herrich)(üchtige naturen, die dermaḱben für ibren Ebrgeiz, um ibre Mad)t zittern, dak [ie 
nur einen Partner [uchen, vor dellen überlegenheit fie licher zu fein glauben, der (ich immer unterzuordnen [cheint. Es bandelt (ich) bierbei keinteswegs blok um wertlole Ehrgeizlinge, fondern die Belelfenheit von die[em Streben nach Macht ift in un[erer Rultur ein allgemein vorherrichender Zug, dellen ungemelfenen Schaden für die Entwicklung der ganzen Men[chbeit die For[chung der Individualplychologie in leinen beiläufigen Grenzen aufgezeigt hat. Wollte man zum Beilpiel das Liebesleben Goethes in die[er Richtung überpriffen, fo würde man mit Staunen auf die auberordentliche Unlicherbeit [token, in die diejer ebrgeizige Mann in leinen Liebesfragen ge= raten i[t.

Wir können lo jene Merkwürdigkeiten ver[tehen, die uns ofters im [eben begegnen, dak Men[d]en in ibrer [iebeswah] in ein viel tieferes und ungeeignetes loziales milieu binab= [teigen. Man findet es zum Beifpiel nicht zu lelten an, dáb ein Mann, der (ich) nur mit den böch [ten Fragen der Men[(b)= heit be[chäftigt, die Mitwelt damit überraloht, daj er etwa leine Röchin heiratet. (tlir, die wir die Gleidbwertigkeit der menlchen lo [ehr betonen, lind darüber nicht etwa entrü[tet, aber wir [ehen hier ein aus der Hrt fallendes Uerbalten und wollen es vom Standpunkte des 5 andelnden veriteben lernen, indem wir leine Endab[icht erforlchen. Uns [c)webt als norm vor, das [ich Menlchen finden, die [ozial und kraft ibrer Uorbildung und Cebensvorbereitung mebr zu einander palfen. Sehen wir uns die Ciebeswerber an, die eine $\langle(J a h)$ getroffen haben, welche von der allgemein erwarteten $a b=$ weid)t, ${ }_{0}$ finden wir in den meilten Fällen Menlchen, die dem Ciebesproblem aujerordentlic) zaghaft und mit Uor= urteilen gegenüber[tehen, vor dem ge[chlechtlichen Partner Furcht empfinden und deshalb einen Partner (uchen, bei dem [ie weniger Macht und Kraft vermuten. Es i[t wobl möglich, daßj jemand aus dem Gefiibl von Stärke von der beiläufigen norm abweid)t. UJir lehen aber in den meilten Fällen, daß es aus $\$$ d)wäche ge(d)ieht.

Somit [cheint eine [old)e (Uab) manchen vor[id)tigen naturen ein aufferordentlid) glüdklicher Griff zu [ein, wenn= 
gleich (ie ibre Endablicht nicht ver[teben, ibre tieferen Beweg. gründe mit Liebe und Erotik überdecken und überzeugt lind, dab bier nur Gott Hmor leine Fand im Spiele babe. Der Uerlauf derartiger Beziebungen ift aber in der Regel (d)led)t. Es erweilt lich, dak diele Art, einer konkurrenz der Ge= (chlechter auszuweichen, mit mannigfachen nachteilen be= haftet i[t. Die Nachteile ent[tehen nicht etwa daraus, daj der intellektuell oder lozial böheritebende enttäu[d)t würde, oder, daj (ich) Schwierigkeiten gelellichaftlicher natur ein|tellen, wenn der "niedrigere" Partner gewillen Forderungen nicht genügt und lo Schwierigkeiten in das Familien = und be[ell= [chaftsleben bringt. Diele und andere äujere Faktoren wären eliminierbar und überbrückbar, wenn (ich) auch) die Endablicht des böberltebenden Partners verwirklichen lallen könnte. Hber es ergibt (ich) die merkwürdige (at|jache; daj der auf der tieferen Stufe [tehende Partner es nicht lange verträgt, leine S(b)äche mißjbraucht zu lehen. (Jenn er auch nicht veríteht, um was es (ich) bandelt, 10 wird er doch das $6 e=$ fübl nicht los, daj hier mit [einer Mangelbaftigkeit ein Unfug getrieben wurde. Er [doreitet aus die[em Gefüb] beraus zu einer Hrt Rache, er wird zu zeigen verluchen, daj er nicht weniger ift wie der andere.

Huffällige Fälle diejer Hrt gibt es viele. Oft wirft (ich ein junges, kultiviertes, an Geift bervorragendes mädd)en in die Hrme eines unbedeutenden, oft logar la[terbaften menichen, vielleicht manchmal mit der Idee, den zu retten, den fie zu lieben lcheint, ibn den Klauen des Hlkobolismus, des Spielteufels, der Indolenz zu entreifen. Doch nie find lolche Men/chen durch Liebe gerettet worden, - die Hktion mißjlingt falt regelmäßjig. Der tiefer[tehende $\tau$ eil Ipürt unter allen Zum[tänden die Bedrückung, die daraus erwäch] [t, daj er niedriger klaflifiziert wird. Er läßst (ich nicht lieben und nid)t retten, weil die bewegenden kräfte leiner Cebenshaltung ganz andere und dem gewöbnlichen Ueritande, dem „common sense", nicht erkennbar (ind. $E r$ bat vielleicht (chon länglt die $5 o f f n u n g$ aufgegeben, dab aus ihm noch etwas werden könnte und lieht in jeder Situation, die ibn als mitmen[ch 
bean(prud)t, eine neue Gefabr, in der leine vermeintliche minderwertigkeit deutlich zutage treten könnte.

Wir kennen auch eine grofse Hnzahl Men[chen, die eine anderen Betrachtungen unerklärliche neigung gerade nur für körperlid) mangelhafte Liebespartner baben. Es gibt junge mädchen, die nur für alte Männer [d)wärmen, und genau 10 bäufig finden (ich) die umgekebrten Möglichkeiten. Diele Cat[achen fallen mit Recht auf und reizen unjer Erklärungs= bedürnis. Sehen wir uns dann die einzelnen menichen an, lo finden wir wobl mandimal eine in bejonders gearteten Uerbältniflen begründete natürliche Erklärung, immer aber entipricht diele neigung auch dem lonitigen Cebensitil dieler menichen, die cinie des geringlten widerltandes einzu[chlagen.

zuir finden ferner Menichen, die falt nur zu folchen Partnern Liebesneigung zeigen, die bereits vergeben find. Diele merkwürdige Catlache kann ver[chiedene Hblichten ver= raten. Sie kann unter Um[tänden loviel bedeuten wie ein "Dein" auf die Forderungen der Liebe, Unmögliches anzu= [treben, zuweilen ein unerfüllbares Ideal. Sie kant aber aud) den Zug des "Udegnebmenwollens" verraten, der von manchen men[chen in die Erotik bineingetragen wird und durch ibren con[tigen Cebens[til determiniert ift. tuir wollen zuerit jene Unzahl von fällen betrad)ten, in denen der Liebeswerber gewillt i[t, der eroti[chen Lebensaufgabe auszuweichen und es auf diefem nicht mebr ungewöbnlichen tJege ver[udht.

Es gibt Men[chen, die für irgend jemand ge[chwärmt haben, obne zul willen, ob er überbaupt exiltiert. Diele baltung (pricht deutlich die Endablicht aus: [ie wollen von Liebe und Ghe überbaupt nichts wil[en und (c)wärmen dort, wo ibre $\$$ (b)wärmerei aller (Ulabr[cheinlichkeit nach niemals realifiert werden kann. Das Gleiche gilt in der überwiegenden Mebrzabl aller Fälle von der dauernd unglü cklich en riebe. Sie ift in den meilten Fällen das mittel, das zu realifieren, was von vorneberein das [ebensziel war: (ich) vom Leben, von der Welt, nun mit einem Sd)ein von Recht, abzukebren. In diejen Fällen kann eine unglückliche Liebe 
gar nicht unglücklich genug [ein, [oll lie die[en Zweck erfüllen. Sie trifft jene Perlonen, die immer [chon am Sprung waren, vor den Fragen des Lebens und belonders vor der Liebe Reibaus zu nebmen. Diele Bereit[chaft zum Reifausnebmen erfährt mand)mal durch einen Cridk, einen Kunitgriff eine willkommene Ueritärkung. Diejer i[t nicht immer aus der Cuft gegriffen, [ondern beftet (ich) an irgendeine rid)tige $\mathrm{Be}=$ ziebung des Cebens und lieht nunmehr gar nicht wie ein Kun|tgriff aus, londern gleicht dem [elb[tver[tändlichen $\mathrm{Er}=$ gebnis einer Erfahrung. Ungeheuer viel Men[chen find für die Gelellichaft noch nicht ganz reif geworden, erblicken in den Liebes= und Ehebeziehungen eine Gefabrenzone und bringen ibre unreife $\mathrm{An}$ (icht in mannigfacher, aber äukerlich) oft unver[tändlicher tueile zum Husdruck. Бört man lie über diele [ie [tändig bedrängenden Fragen (ich) äu仍ern, fo ver= nimmt man Allgemeinheiten, die wobl in irgend einem Zulammenhang wahr lein können und nicht tJindbeutelei bedeuten müllen. WUenn zum Beilpiel ein auch an[on[ten Zaghafter meint, er beirate nicht, "denn das Ceben lei jettt 10 [chwer", lo ift wohl jedes (Ulort wahr für die, die nicht beiraten, aber gleichzeitig auch für die, die beiraten. Diele Uabrbeiten werden aber nur von jenen geäu\{bert, die auch obne dieje (Ulabrheiten "Mein" gelagt bätten, nur bätten lie dann andere "Wahrbeiten" aufgegriffen. Es wäre nicht diplomati[ch, eine vorgefalste Hblicht mit (c)lechten Gründen Itiitsen zu wollen, wenn gute Gründe überall zu baben lind. Wer Gelegenheit gehabt hat, [ich von der er[chreckenden நäufigkeit jenes cypus von Menichen zu überzeugen, der vor der Löfung von Cebensfragen Reifaus nimmt, wird (ich) über die Einkleidung dieles Zuges in die Erotik nicht wundern.

Für Husreiber ift ein kunitgriff bejonders zu empfehlen, der vielfach erprobt i[t. Man (chaffe (ich) eine neue Idee, ein belonderes Ideal. An dielem Ideal melle man nun alle men: [chen, die einem über den Lebensweg laufen. Dies hat zur Folge, dak lich keiner als geeignet erweilt. Hlle weichen vom Ideal $a b$, und wenn wir (ie ablebnen und aus[chalten, 
1o fieht unler Cun nur vernünftig und woblbegründet aus. Erit wenn wir den Einzelfall berausnehmen und betrachten, Io finden wir, dajs die 10 vernünftig wäblenden Men(c)en audb obne ibr Ideal von vorneberein gewillt waren, "Dein" zu [agen. Im Ideal finden (ich) wünichenswerte Ziele von 0ffen= beit, ZUabrbeitsliebe, Mut u[w. Sie [tellen Begriffe dar, die wir nach unlerem Belieben io lange dehnen und [trecken können, bis [ie jedes men[(b)liche Mak über[chreiten. Udir baben es daber in der நand, uns etwas zu wün[chen, das wir vor= ber unerreichbar "gemacht" haben.

Der Kun[tgriff, dadurch nicht lieben zu mü[len, dajs man etwas Unerreichbares liebt, findet ver\{chiedene Möglichkeiten der konkretilierung. Ulir können eimen Menlchen lieben, der einmal eine kurze Zeit da war, Eindruck machte, ver= (c)wand und nun nicht mebr zu finden i[t. Man müßjte die ganze (delt durchluchen, um ibn zu finden. Im eriten Moment werden wir gerübrt, went wir von einer 10 innigen und treuen Ciebe bören. Die Bedingung aber, die bier der Uerwirklichung der Ciebe auf Erden ge[tellt i[t, nämlich) die ganze (Uelt zu durch)[uchen, um ibn zu finden, ift übermen(c)lid) und fordert unjeren bereits geweckten Argwobn beraus.

(Uir können uns auch einen men[(h)en unerreichbar "machen". Oft bat der Liebeswerber [dhon im Hnfang leiner ulerbung den Eindruck, daj er bier nicht gut ankommen werde. Dięer um[tand wird logleich der Husgangspunkt einer größseren Hktion. Er glaubt, ohne die geliebte Perfon nicht leben zu können, bewirbt (ich) um [ie, obwob] jeder objektive Zu[eber es für unwabricheinlich balten müßste, daßj er jemals Gegenliebe finden werde. Er lagt es (ich auch lelb[t. Oft kant man auch beobachten, dá [oldhe Bewerbungen in einer Form gelcheben, die für (ich) allein (chon im[tande i[t, den videripruch des anderen berauszufordern, indem [ie z. B. auffallend vebement er= folgt oder zu einer Zeit, in der irgendweldbe Garantien für ein Zu[ammenleben nod) gar nicht möglich) und gegeben find.

Derlei Bewerbungen lind abgezielt a uf unglü dkli the Liebe. Die Unzabl von Men/chen, die bei ibren Bewerbungen auf das Ziel: unglückliche liebe zu[teuern, i[t geradezu über= 
ra[chend. Man (ollte doch denken, wenn man an diele Dinge von auksen berantritt, ein [oldhes Uerbalten liege gar nicht in der men[thlichen natur. (Jir [ehen es aber diejen Menichen von

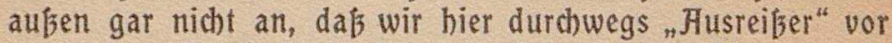
uns haben. Die individualp\{ychologi[(h) Durchleuchtung macht dann klar, daßj eine unglü ckliche Liebe für dieje Hrt von menjad)en ein ausgezeichnetes Uer[teck bedeutei. Denn wenn ein Unglück= licher [eine unglüdkliche Liebe fünf oder zehn Jabre mit (ich) berumträgt, fo ift er eigentlich während diejer ganzen Zeit vor allen anderen [ölungen die[er Frage in Sicherbeit. $E r$ hat woh] gelitten, hat für die Durchletsung [einer Hb(icht Rolten erbrach)t, aber lein Ziel, das ihm lelbit unbewujit blieb, das er lelbit nicht ver[tand, nämlich (ich) von der Cölung der Liebes= und Ehefrage fernzubalten, bat er damit bei gutem Gewillen und gerechtfertigt völlig erreicht. Daß dieles Ziel und diele leine Lölung, die eigentlich keine Lölung i[t, (ich mit den bege= benheiten unjerer Erde und der Logik des men[chlichen Zu= [ammenlebens nicht verträgt, ift eigentlich leine tief\{te Cragik und erft diele lette tieffte Einfid)t vermag bier korrigierend einzugreifen.

Liebesneigungen zu Menlchen, die bereits eine andere eroti[che $t(J a b]$ getroffen baben, mül[en nicht immer ein "nein" be deuten. Die Ge[chichte bedeutender Men[ahen kann uns darüber belebren, dafs die Men[chen in unjerer fo komplizierten kultur mit einer aukerordentlichen Begierde des Raubens, des tUeg= nebmens heranwach (en. Das (Jerlangen nath) verbeirateten Frauen hat immer ermeute Hiktionen im Gefolge, (ich des Liebesob= jektes zu bemächtigen. wenn diele Hktionen auch äukerlich oft die edel[te form wahren. Einer diefer Cypen (c)eint Ridbard zJagner gewelen zu lein, in delfen dichteri\{che Schöpfungen fajt immer der sinn eingewoben, die Komplikation bineinge= tragen i[t, dał der Feld eine Frau begel)rt, die (c)on zu einem anderen gebört. Huch) das Ceben Richard (Ulagners zeigt diele Cinie.

Hllgemein be[timmt das Un[icherheitsgefüh] viele Formen der Erotik. Es gibt junge Männer, die nur für ältere Frauen sympathie empfinden, in irgendeiner irrtümlichen Meinung, 
dak bier die Scbwierigkeiten des Zulammenlebens geringer wären. Huch verraten [ie ibr Gefüb] der Schwäche durch ein gewilles Bedürfnis nach mütterlidber Für[orge, (ie gebören zu den meilt verzärtelten Menlchen mit grobem Anlehnungsbe= dïrfnis, von denen man lagt, dab [ie "noch eine Hmme brauthen". Sie bereidhern jenen $\tau$ ypus, der dem anderen Ge(chlecht gegen= über nie genug sicherungen baben kann, und in böchite Unrube verleht wird, wenn er dem anderen Ge[chlecht gegen= über[teht. In unlerer Kultur gibt es eine erichreckend grofje Zabl [olcher unlicherer Men[chen, lie tragen den Schönbeits= fehler unjerer Entwicklungsphale [tark ausgeprägt mit [ich: die Furcht vor fiebe und $\in$ be. Dies ilt keine Husnabme= erfocheinung, londern ein allgemeiner Zug der Zeit. Es wim= melt in unferer Gejell[chaft von Husreifern. Sie lind aus irgend einer unglücklichen und irrigen Stellungnabme heraus immer wie auf der Flucht, kommen (ich) immer wie gejagt und verfolgt vor. Es gibt Männer, die (id) ifolieren und ver[tecken, es gibt Mädchen, die (ich gar nicht mebr auf die Galle trauen, überzeugt, daf (ich) alle männer um lie bewerben, und daj fie immer nur das Objekt von Angriffen wären. Gier treibt die reine Eitelkeit ibr Spiel und vermag das Leben eines menlchen oft völlig zu verpiulchen.

Hus Erfabrungen und Erkenntnilien laflen (ich gute und (chlechte Dutanwendungen zieben. Ulir finden unter den (c)led)ten Nutsanwendungen die übertriebene Umkehr eines Fehlers, welche eben[o ein Febler i[t. Das Gegenteil von Zurüdkbaltung und Uer[b)loffenheit i[t Offenbeit, und 10 finden wir Men/chen, die durch Offenbeit Fehler begeben. Es gibt men[chen, die immer die neigung zeigen, (ich anderen a n $\mathrm{z} u=$ bieten. (Jenn es au(h) lehr [chön ift, leine liebe offen zu bekennen, find wir trotsdem ebenlo tief von der (Ulabrheit durchdrungen, dajs man damit in unierer nicht einfach zu nebmenden Kultur einen [d)weren Fehler madht. Es gibt nämlich keinen Menj(chen, der diefes Anerbieten glatt vertragen wïrde, und der Uoreilige wird dann nicht nur jelbjt den Schmerz der Reue und die Lalt auftretender 5emmungen zu tragen baben, [ondern auch den Partner in der unbefangenen 
Entfaltung leiner Liebesregungen [tören, weil diẹer, bei dem allgemein herr\{chenden Miß 5 brauch, der mit der Liebe getrieben wird, bei der beftehenden Spannung und dem Rampf der (Ge[d)lechter untereinander, nie recht im klaren lein wird, ob das Anerbieten echt und wabr geweien i[t oder ob nicht etwa [chlechtere Hblichten (ich dabinter verbergen. Es gibt keine fe[ten Geletse, - wir haben die Hufgabe, die Eigen= heit des Parmers in Recbnung zu ziehen und uns an die Gegebenheiten unjerer Kultur zu halten. Es [cheint heute eher empfehlenswert, feiner neigung ein wenig die Zïgel anzulegen.

Eine be[ondere Rolle [pielt die Ciebe, [owobl die glück. liche als auch, und in böberem Makse, die unglückliche Liebe bei Kün[tlern. ZJir können wohl lagen, dak die unglück= liche Liebe eine 10 allgemeine Zeiter[cheinung i[t, dak es kaum einen geben dïrfte, der nicht einmal von ibr und ibren leelifhen Be[chwerniflen heimgelucht worden wäre. Unter den Men[chen aber, die mit belonderer Empfindlichkeit dem Leben gegenüber[teben, [pielt der kün[tler eine aufjerordentlich) pro= minente Rolle. Er wird [ction dadurch auffällig, dajs er in leiner Kunit ein Leben "neben dem [eben" (ucht, (ich nicht in der Realität betätigt, [ondern eine Er[atswelt [ucht, von der (Jirklichkeit nabezu abge[topen wird, freilich aber er[t dann zum künftler wird, wenn er leine Scböpfungen 10 ges [taltet, dak [ie der wirklichen (Uelt förderlich werden. Jedes Kunftwerk ift er[t dadurch ein kun[twerk, da仍 es allgemein[ten tuert befitst, daj der kün[t]er in [einer schöpfung den tueg zur (Uirklichkeit und zur Gemein[chaft zurïckfand.

In der Hbbiegung aus dem realen Ceben liegt ein Zug, die Realität des Cebens betonende Einrichtung der Liebe und Ehe als feindlich) und (törend zu empfinden. (Ulir begegnen einer Unzah] von Kün[tlern, die die Bindungen des Cebens wörtlich als Bindungen, Felfelungen, als Findernille auffallen und lie logar in ibrer Phantafie máklos ausgeftalten. Sie kommen - über diefe nun als máblos empfundenen Findernilfe kaum hinweg, befinden (ich in ihren Ciebesbeziehungen vor einer unlösbaren Hufgabe und zeigen hierbei nicht nur die $\mathrm{Be}=$ wegungen eines Ciebenden, [ondern gleich zeitig und in noch) 
viel [tärkerem Make die Bewegungen eines Menlchen, der vor der Ciebe die Fludt ergreift. Dies drückt (id) in ibren Gedanken und Schöpfungen aus, die die Probleme der Men/chen in unter[trichener Form wideripiegeln. Der anders= geidblechtliche Partner wird in irgendeiner tueile als über= mächtig empfunden und bald erhält die Liebesiphäre den Ebarakter einer Gefabr. Dielen Gedanken findet man falt wörtlich in mitteilungen von Dichtern und S(briftitellern. Flle problematilchen naturen baben den gleichen Zug, weil lie alle aujerordentlich ehrgeizig und empfindlich find und jede Beeinträchtigung ihrer Machtvollkommenbeit als eine [chwere Beleidigung oder eben als Gefahr auffallen. So lagt der Didter Baudelaire: "Ich habe nie den Gedanken an eine [chöne Frau fallen können, obne gleichzeitig die Emptindung einer ungeheuren Gefabr zu baben."

Der Anblick eines Menidhen, der nun einmal die von ibm vermeintliche "Gefabrenzone" betreten hat, bietet uns eine Hufeinanderfolge von Hbwebr= und Sicherungsbewegungen. bebbel [childert in einem Briefe, den er als Jüngling leinem Freund lendet, leine Empfindungen ungefäbr folgendermajen: "Datürlich wobne ich hier [chon wieder dem [chön[ten Mädd)en der Stadt gegenüber und bin bis über die Obren in fie ver= liebt; aber boffentlich wird (ich auch hier bald das Begen= gift neben dem Gift finden .... Und wenn ich heute noch lebe, wie ibr Geliebter, zu ibr durd)s Fen[ter [teigt, [0 bin id) mit ibr fertig." Dies ift das Ergebnis eines Eindruckes, von dem wir eigentlich einen anderen Husgang erwartet bätten.

Die Frau als Gefahr ift ein dauerndes Ceitideal der Kun[t. Man durd)multere die Bilder des Malers Rops, die die Frau als Gefabr, als Schrecken oder zumindeit als ungeheure Macht dar[tellen. Die Kun[t i[t beute vorwiegend eine männliche Kun[t, (ie trägt in (ich) die männliche Cradition, bringt vorwiegend männliche Probleme und bebt die Frau zu jener Zauber= oder Schreckge[talt empor, die [ie in den Hugen vieler Männer i[t. Die Frauen können bei dielem männlichen Zeitideal nicht mithalten und finden in der Husübung der kun[t Schwierigkeiten, nicht weil lie nicht fähig wären, [ondern weil lie dem übertriebenen 
männlichen Ideal nicht dien[tbar \{ein können. Die Ulorrede zu "Caulend und eine Racht" zeigt uns, wie der Hutor be= fonders er[chrocken auf die cift und Ver[chlagenheit der Frau binweift, die durch eine unglaubliche Erfindungsgabe dem Manne gegenüber ibr Ceben rettet. Hber auch die älteiten Gebilde der kunft, zum Beilpiel die Bibel, die ja alle menlchen von den er[ten Kindheitstagen mit der ibr eigen= artigen Stimmung erfa[jt, ift von dem [tändig begleitenden Gedanken durchdrungen, dajs die Frau eine Gefahr voritelle, lo dab das Kind in Schüchternbeit, Unausgeglichenbeit, Zag= baftigkeit dem tueibe gegenüber aufwäch[t. Eines der größsten Kun|twerke, die Iliade, malt mit grofjer Präzifion das Ulnglüick aus, das durch eine Frau angerichtet wurde. In allen Dicht= werken, in allen Run|ftwerken tönt das Problem der Zeit: Die Frau als Gefabr. Grillparzer lagt von (ich) „Uor der Liebe babe ich mich in die kunit gerettet."

tdir können nicht obneweiters voraus[agen, wie eine un= glückliche neigung in einem men[chen (ich) auswirken wird. Seine Ge[amtlebenshaltung, leine Lebenslinie ift hierfïr maßj= gebend. 5aben wir einen Menldhen vor uns, welcher bei auf= tretenden \$chwierigkeiten den Mut verliert und leine Aktivität abbricht, 10 wird auch der Schiffbruch) in der Liebe ibm Schiff= bruch des Cebens [ein köntten. In der unglücklichen Liebe jelbit ift diele Konlequenz $\mathrm{ni}$ ich $\mathrm{t}$ enthalten. Derjenige, dem der Plan innewohnt, durch Schwierigkeiten aufge\{tachelt zu werden, wird (ich aus einer unglüdklichen Ciebe aufraffen und zu groben Ceiftungen gelangen. Die ungliidkliche Liebe ilt weder Tragik noch beilmittel, beide konjequenzen werden aus ibr gezogen werden können, je nachdem, ob die Konlequenz von einem mutigen oder einem niedergebrochenen menichen gezogen wird. Die vulgäre Plychologie weilt öfters auf die groben Leiltungen hin, die aus einer unglücklichen riebe entitehen. Sie empfieblt [ie manchmal als Medikament. (Jir kennen Menl(hen, die auch obne unglückliche Ciebe Grofjes geleiltet haben. Der rid)= tige Kern diejer halben UJabrheit ilt, daj der kün[tler vom Problem der Liebe in ganz belonderem Maje gepackt und gefellelt wird. 
Das Ceben Goethes ift in dieler Finficht belonders lehr= reich. Immer erblickte er in der Frau die Gefabr, immer war er vor ibr und vor der fiebe auf der Fludt)t. Die Leitlinie des "Fault" ilt ein ewiges Sudben nach einer cölung des Liebesproblems. Mit leinen eigenen Spannungen und Regun= gen und Strebungen baute er leine Wlelt, unzufrieden mit den Catlachen des Cebens, und zauberte allgemein Men(d)liches vor un[ere Hugen. Es madht die Größje leiner Kunjt aus, dab alle Seiten in uns mitklingen, wenn er das ewig neue Lied der Spannung der Gelchlechter ertönen läßjt, in die befangen die Men[chen befürchten, dabs bingabe gleidbedeutend [ei mit Uer. lu[t der Per[önlichkeit, Förigkeit oder Sklaverei.

Erwähnt (ei bier nod) $s$ chleiermacher, der in einer wundervollen Hbbandlung zu erweilen (ucht, daj Liebe gar

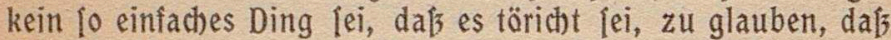
der Men[(h), wenn er ins Ceben binaustritt, auch (b)on etwas von Liebe ver[tehe. Es mülle eigentlich jeder eine gewille Uor= übung, eine Vor[chule leichterer Hrt durchmachen. Huch dieler Idealift reiniten tual[ers, von den religiö[e[ten men[(h)en boch)= verehrt, kann (ich der (uberzeugung nicht ver[chließsen, daßj die Menloben in der fiebe nicht lo leicht zueinander finden.

In meinen Uorlejungen über Men[chenkenntnis, die [tändig von zirka 500 Perfonen belucht werden, gelangen gröften= teils Fragen über Ciebe an mich, als ein Zeichen, wie (ich) die Menjhen in diefer Frage weniger leicht zurecht finden, als in denen des Berufs etwa.

ZJarum es to wenig glückliche Liebesbeziebungen gibt? zuir find noch nicht die richtigen Men[then, wir (ind noch) nicht reif zur Liebe, weil wir mit der Mitmen[(h)lid)keit im Rück= ftande [ind. UJir webren uns mit allen Mitteln, weil wir uns zu viel fürchten. Man bedenke nur, auf welche Schwierigkeiten der Gedanke der gemein[amen Erziebung der Ge(b)lechter, der Roedukation, [tößst, welcher ja doch nichts anderes bezweckt, als daj die Gelchlechter frühzeitig ibre Scheu und Hngit ver= lieren und bereits von Jugend an Gelegenbeit baben, (id) belfer kennen zu lernen.

Für Schwierigkeiten in den Liebesbeziehungen gibt es kein 
beilmittel in Form einer fe(ten Jerhaltungsregel. Die Erfah)= rungen der Individualp[ychologie zeigen es täglich aufs neue, dáb die Hb[onderlichkeit der Erotik eines Men[chen ein Schnör= $\mathrm{kel}$ [einer Ge[amtper[önlichkeit i[t, die es in jedem Einzelfalle zu erfallen gilt. Udir muillen den Zulammenbang aller Ḧ̈uße= rungen eines Men[chen erfal[en, [eine Per[önlichkeit und deren Beziehung zur Ulmwelt ändern, um leine fehlgegangene Erotik zu verändern. Die Bewegungslinie eines Men[(h)en wird (id) auch) in der liebe geltend machen. Sie wird ibn zwingen, einmal eine unglückliche ciebe zu [uchen und darin zu ver= harren oder lie wird ihn diele leichter nebmen lalfen und ibn zum Hufftieg führen. Sind es Menlchen, die, mit Ehrgeiz vollge[ättigt, eine Uerweigerung irgendwelcher Hrt nicht zu er= tragen vermögen, 10 wird aus die[em, in einem Ge[amtzu= [ammenbang aufklärbaren Irrtum heraus der Selbitmord nabe= liegend er[cheinen und in un[erer, die Unterordnung gebieten= den Ge[el![ (h) aft eine Gelegentheit zu einer bochtragi[chen Situation bieten, mit der Flucht aus dem reben die Rache gegen die Gelellichaft und gegen einzelne Perjonen zu verbinden.

Die Liebe kultiviert [id), und die Beziehungen der Liebe ver[chönern und verfeinern (ich mit der kultivierung und Ent= wicklung des alle Men[chen umfallenden Gemein[chaftsgefühles. Die Beziehungen der Ciebe ge[talten (ich nicht plötlich, fondern zeigen eine lange Uorbereitung. Die erotilche Bindung ift zwilchen den Menlchen immer vorbanden, es bedarf aber ge= willer Bedingungen, um lie als Liebe fühlbar und lichtbar zu machen. Der Beginn von Liebesregungen reicht zu= rück bis in jene fernen (age, wo (ie nod) nicht Erotik, noch nicht lexuell gefärbt gewelen [ind, londern wo (ich) noch der breite Strom des Gemein[(h)aftsgefübls in den Formen der Hn= bänglichkeit und Zärtlichkeit ergof und einzig und allein nur jene allgemein men[chliche Beziehung lichtbar war, die (wie zwi= (chen Mutter und Kind) die Men[chen lofort verbindet, obne dafs (id) jenes dauerhafte der Ewigkeit und dem Be[tand der Men[(h)= beit dienende Band unter einzelnen bildet, das wir die cie be nennen. Sie iff Band und Uerewigung zugleich. Diele Bezie= bungen kann man nicht eigenwillig ge[talten, muj vielmehr 
ibre Huswirkung zulallen. Das tuillen um diele Dingevilt noch) nicht reif, weil der Men[ch fähig i[t, (iic) über die Uore gänge in leiner eigenen Seele zu täu[chen. Beide Geichlechter geraten nur zu leicht in den Strudel der Preltigepolitik und [pielen dann eine Rolle, der lie beide nicht gewachlen [ind, die dazu führt, die 5armloligkeit und Unbefangenbeit ihres Lebens zu [tören und lie mit Ulorurteilen zu lättigen, denen gegenüber natürlich jede Spur wahrbafter Freude und jedes Glücksgefüh) ver[कwinden muk.

tuer diele Gedanken in (ich aufgenommen hat, wird zwar natïlich nicht fehlerlos auf Erden wandeln, (iid) aber wenig. ftens des rechten tJeges bewu[jt bleiben und leine Irrtümer, ftatt lie zu vergröbern, [tändig verkleinern können. 

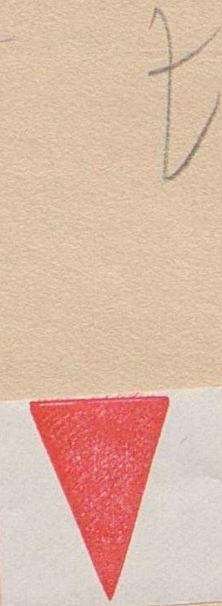

$380 / 80 / 40569(2)$

$\times 1368040569200019$ 


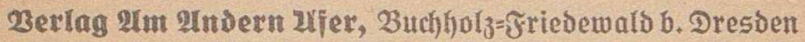

G3opuläre Riferafur Det Sttbivioualpinchologie

\section{Gchwer ersiehbare Sinder}

Eine Sthriffenfolge

\section{Serausgegeben non \\ DIt und afice 2 if}

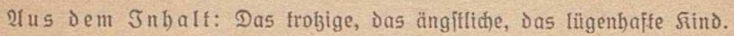

Breis des Bändchens S $-.90(\mathrm{Mk},-.60)$

Berlag voa Matin 2Barnecti, Berlin

\section{Du und Der MIIfag}

Eine भुjuchologie des fäglichen Bebens, in Berbinbung mif Ireutuen ber Snbivibualpindjologie

Serausgegeben von

अohannes शteนmanก

Breis elma S 9.- (Mk. 6.-)

2f us Dem Snbalt: I. Die 2fufgaben Des 2tllfags. II. Der Gpiegel Des थlltags. III. Der Gdtüfifel zum थilfag.

23 erIag von $\pi$.

\section{Die (Bemeinichail}

\section{Miffeilungsblatf}

Des Internafionalen Bereines fïr Snbivibualpinchologie Serausgegeben von

भida Beil

2bonnementspreis: S 4.30 (Mk. 2,70)

3u bezietien Durch die Bubhandung

Morib Berles, Mien, I., Geilergalie 4 


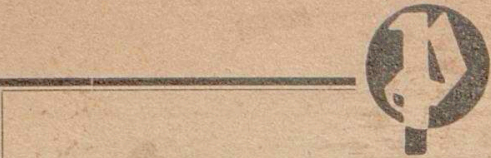

Verlag von Moriz Perles, Wien, T., Seilergarle 4

\section{Richtige Cebensfiibrung}

Uolkstümliche Huffätse zur Erziehung des Menichen

nach) den Grund(ätsen der Individualplychologie

In diejer von sofie Lazarsfeld herausgegebenen

\section{5ammitum}

find folgende befte erfchienen:

Jom bäuslichen Frieden von

Sofie Cazarsfeld

Seelitehe Entwicklungs:

bemmangen

Dr. Erwin tUexberg
Geburtenregelung von

margret bilferding

Die Sprache des Rindes und ihre. Fehler

Dr. Ceopold Stein

In Uorbereitung befinden (ich:

Begabung und Invegabtheit

Dr. Hlfred Hdler

Elternilieve

von

Filde Krampflitichek

Die welt des Rindes

Gina Kaus

Schönheit und Pertönlichkeit von

Dr. Robert Lazarsfeld
Erziehung zur Ehe ven

Sofie Lazarsfeld

Cechnik der Erziehung Ida Löwy

schule und Familie von

நugo Lukacz

Soll man strafen? von Sulanne Schalif

Preis jedes Fieftes $80 \mathrm{~g}=50 \mathrm{Pf}$.

Durch alle buchbandlungen zu beziehen 

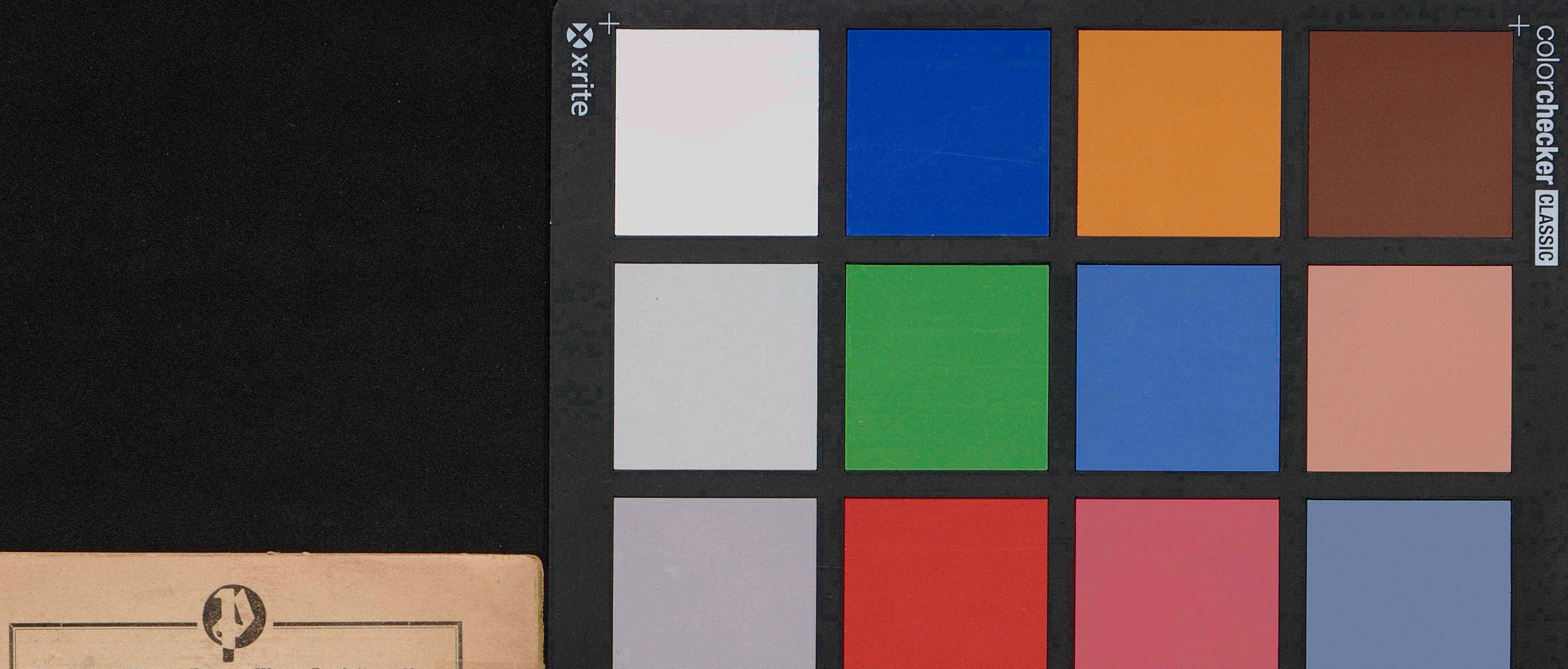

Uerlag von moritz Perles, wuien, T., Seilergafre 4

\section{Richtige Lebensfiubrung}

Uolkstümliche Huffätse zur Erziehung des Menlchen nach den Grund/ätsen der Individualplychologie

In diefer von sofie Lazarsfeld herausgegebenen 5ammlumg

find folgende Fefte er[d)ienen

Uom bäusliehen Frieden

Sofie Lazarsfeld

Seelitche Entwicklungs:

hemmangen

Dr. Erwin Wexberg

In Uorbereitung befinden lich

Begabung

und Invegabtheit

Dr. Hlired Hdler

Elterinliebe

Filde Krampflititek

Die twelt des Kindes

Gina Kaus

Geburtenregelung

margret Filferding

Die Sprache des Kindes und ihre. Fehier

Dr. Ceopold Stein

Erziehung zur Ebe

Sofie Lazarsfeld

Cechnik der Erziehung

$$
\text { Ida Löwy }
$$

Schule und Famille Furan rubart
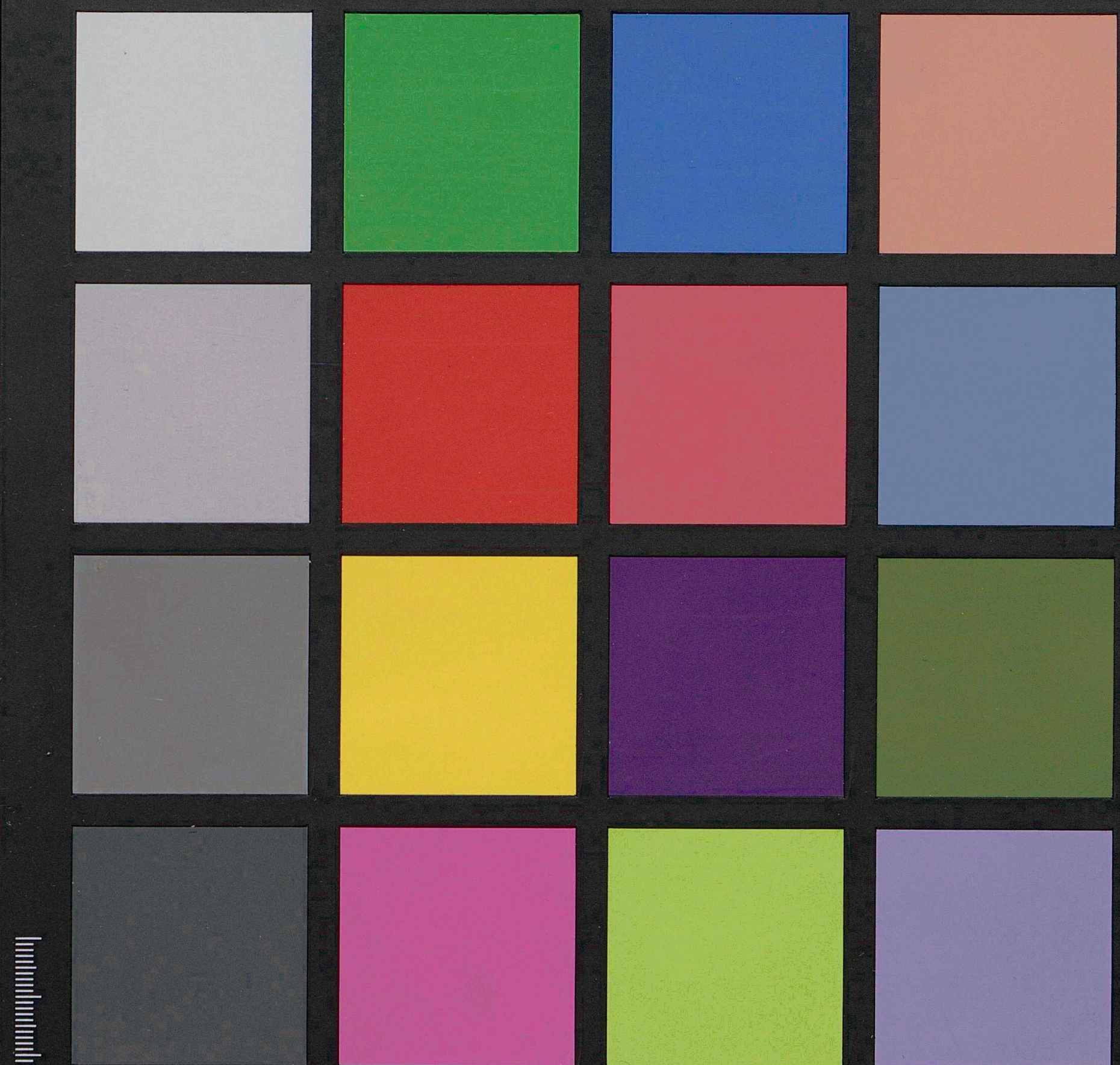

Freie Universität Berlin

Britiber Eollinef, Wien, III. Steingaffe 25.
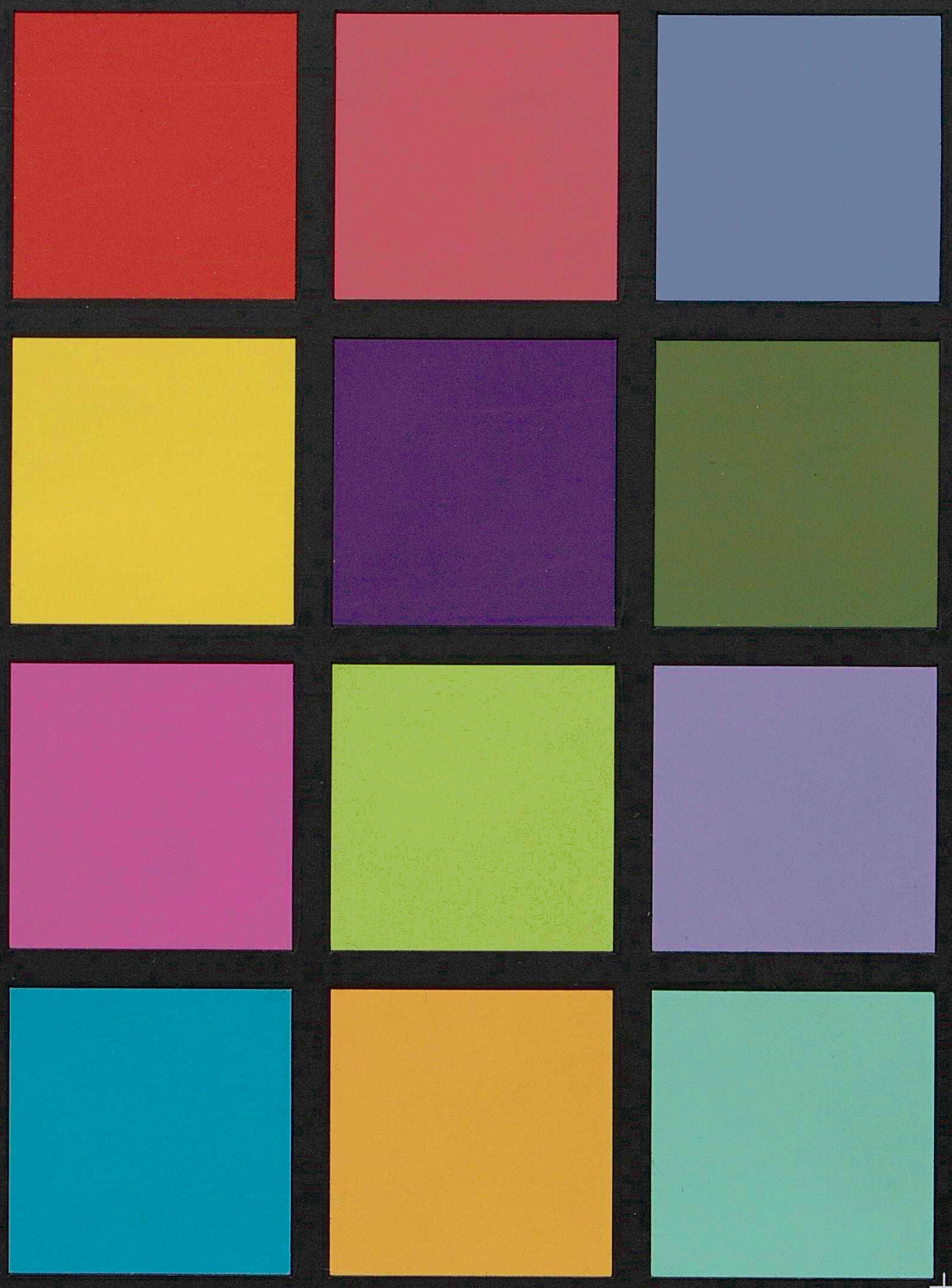